DOI:

$10.1038 / \mathrm{nrm} 2449$

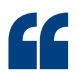

...exciting perspectives

that are

opened up by

the twofold

enhanced

resolution of

3D-SIM...

NUCLEAR ENVELOPE

\title{
The power of two
}

Improving resolution beyond the 200-nm diffraction limit has been a long-standing goal that was achieved in recent years by the development of subdiffraction-resolution imaging techniques. The groups of John Sedat and Heinrich Leonhardt have now used one such technique - threedimensional structured illumination microscopy (3D-SIM) - to study the nuclear periphery in mammalian tissue-culture cells.

Working together, they imaged interphase chromatin at the nuclear periphery using cells that were stained with DAPI (4',6-diamidino2-phenylindole). Remarkably, they observed thousands of well-defined holes in the DAPI staining that were not apparent in the corresponding wide-field epifluorescence images.

To test whether these holes represent the exclusion of DNA from nuclear pore complexes (NPCs), Sedat, Leonhardt and colleagues co-immunostained cells with NPC-specific antibodies and antibodies against lamin B (a major component of the nuclear lamina that lines the nuclear envelope). For comparison, they imaged the samples with confocal laser scanning microscopy (CLSM). Remarkably, 3D-SIM images consistently revealed a triple-layer organization, which was not resolvable by CLSM, in which the peripheral chromatin rim was outlined by a fine layer of the nuclear lamina with nuclear pore signals on the cytoplasmic side. Using an antibody against a nucleoporin that is located on the nucleoplasmic side of the NPC, the NPC signal was in the same plane as the lamin B signal. This further demonstrates the subtle differences that can be detected in the $3 \mathrm{D}$ organization of the nuclear periphery.

Practically every DAPI void contained a focus of NPC staining, which further confirmed that most, if not all, NPCs exclude chromatin. Furthermore, the density of NPC foci was higher as calculated by 3D-SIM than by CLSM. Measurements of the width of the NPC were in good agreement with those obtained using electron microscopy. Finally, 3D-SIM

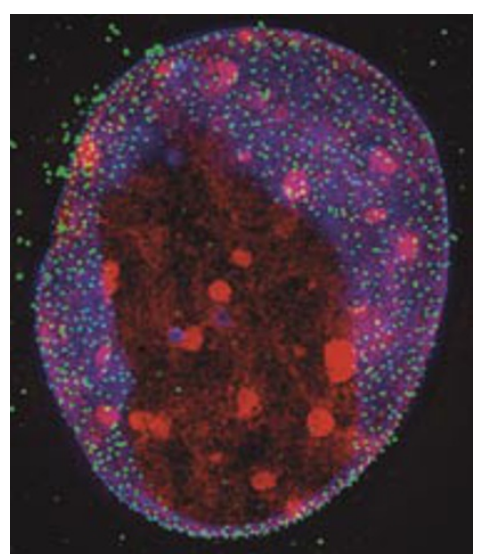

The image shows a nucleus from a mouse myoblast cell; DNA is shown in red, the nuclear lamina in blue and nuclear pores in green. The upper surface of the nucleus has been clipped in this projection to reveal the DNA staining underneath the lamina. Image courtesy of P. M. Carlton, University of California, San Francisco, USA.

allowed invaginations of the nuclear envelope to be resolved as bilaminar structures, which was not possible with CLSM. This study demonstrates the exciting perspectives that are opened up by the twofold enhanced resolution of $3 \mathrm{D}$-SIM in lateral and axial directions over conventional fluorescence imaging techniques.

\section{Arianne Heinrichs}

ORIGINAL RESEARCH PAPER Schermelleh, L. et al. Subdiffraction multicolor imaging of the nuclear periphery with $3 \mathrm{D}$ structured illumination microscopy. Science 320, 1332-1336 (2008) 